\title{
QM in der klinischen Geburtshilfe - sinnvoll?
}

\author{
Karin Schmidt, Hebamme, M. A., Anja Siegle, Kinderkrankenschwester, M. A.
}

\author{
Mit Qualitätsmanagement verfolgt man die gute Absicht, für die Einzelnen das derzeit \\ bestmögliche Behandlungsergebnis zu erreichen. Hierfür werden einige Anstrengungen \\ unternommen: medizinische Fachgesellschaften erarbeiten Leitlinien, Kliniken führen \\ Qualitätsmanagementsysteme ein und lassen sich zertifizieren und der Staat kontrol- \\ liert Krankenhäuser mithilfe von Qualitätsindikatoren. All das kostet viel Zeit, Mühe und \\ Geld. Zu Recht darf deshalb gefragt werden: Was bringen diese Strategien? Haben die \\ Frauen einen Nutzen?
}

Qualität zeigt das Ausmaß, in welchem eine Sach- oder Dienstleistung definierte Erfordernisse erfüllt [1]. Qualität ist somit nicht per se etwas Positives, sondern ist ein Soll-Ist-Vergleich, der negativ oder positiv ausfallen kann. Für die Messung der Qualität einer Sach- oder Dienstleistung werden Kriterien benötigt, die die Anforderungen beschreiben, die man an die jeweilige Leistung stellt.

Es gibt verschiedene Gründe, weshalb es sinnvoll ist, die Qualität von Gesundheitsdienstleistungen $\mathrm{zu}$ sichern und $\mathrm{zu}$ verbessern: 1. Gesundheit ist ein hohes Gut, das nach allen Regeln der Kunst bewahrt und geschützt werden muss [1]. 2. Der Grad an Informiertheit der Menschen ist verhältnismäßig hoch und die Ansprüche an die Versorgung scheinen gestiegen zu sein. 3. Seit 2003 sind alle deutschen Kliniken dazu verpflichtet, die Qualität ihrer Leistungen zu sichern, weiterzuentwickeln und transparent darzustellen (SGB V §135a) [2]. Wie die Kliniken diese Vorgaben umsetzen, ist ihnen selbst überlassen. Daneben fordert das Gesetz, dass die Leistungen „dem anerkannten Stand der medizinischen Erkenntnisse [...] entsprechen und den medizinischen Fortschritt [...] berücksichtigen“"(SGB V §2, §135a). Behandlungen sollen sich auf wissenschaftliche
Studien und klinische Erfahrung stützen [3]. Es sollen also Erkenntnisse aus aktuellen Studien und Übersichtsarbeiten in die tägliche Praxis einfließen, um eine bestmögliche Qualität für die Patientinnen zu erreichen. Dieses Vorgehen nennt man evidenzbasiert.

Nachfolgend werden wichtige Instrumente des Qualitätsmanagements und ihr jeweiliger Nutzen vorgestellt.

\section{Leitlinien}

Leitlinien sind systematisch entwickelte Entscheidungshilfen für Ärztinnen und Ärzte. Im Sinne der Evidenzbasierten Medizin vereinen sie aktuelle wissenschaftliche Erkenntnisse mit praktischer Berufserfahrung [4]. Die medizinischen Leitlinien der Deutschen Gesellschaft für Gynäkologie und Geburtshilfe findet man auf der Internetseite der Arbeitsgemeinschaft der Wissenschaftlichen Medizinischen Fachgesellschaften (www. awmf.de).

Geburtshilflich relevante Leitlinien gibt es zu Themen wie peripartale Blutungen, vaginal-operative Geburten, Terminüberschreitung, die Zusammenarbeit zwischen Hebamme und Arzt. Dabei handelt es sich fast ausschließlich um S1 Leitlinien. S1 Leitlinien sind Empfehlungen einer Expertengruppe, die im informellen Konsens festgelegt wurden [5]. Das ist die niedrigste Evidenzstufe von Leitlinien und darf nicht als unumstößliche Wahrheit missverstanden werden. Diese informelle Konsensfindung ist besonders fehleranfällig und lässt offen, welche Inhalte auf persönlicher Meinung und welche auf wissenschaftlichen Fakten basieren. S1 Leitlinien beruhen nicht auf einer systematischen Suche nach wissenschaftlichen Studien $[5,6]$.

Lediglich die Leitlinien zur Versorgung von Neugeborenen diabetischer Mütter (S2) und zur Behandlung einer Brustentzündung in der Stillzeit (S3) entsprechen einem höheren Evidenzgrad.

Abgesehen von dieser Problematik ist in der geburtshilflichen Versorgung nicht bekannt, in wieweit Leitlinien generell zur verbesserten Versorgung und Betreuung der Frauen beitragen. Es lässt sich nicht belegen, dass ein besseres Outcome für Frauen und Kinder oder ärztliche Verhaltensänderungen auf Leitlinien zurückzuführen sind [7]. Unklar ist, ob dies an den Leitlinien selbst oder an ihrer mangelnden Übertragung in die Praxis liegt. 
In einer Studie von Knoll wurde untersucht, in wieweit ausgewählte Bereiche der Geburtshilfe (Tokolyse, Abort, Weheninduktion, Antibiose bei Sectio) evidenzbasiert sind und Leitlinien folgen. Die Autorin stellt in den Kliniken sehr unterschiedliche Vorgehensweisen fest, die nicht immer dem aktuellen wissenschaftlichen Stand entsprechen [8]. Derzeit lassen sich die großen Hoffnungen, die in evidenzbasierte Leitlinien gesetzt werden, (noch) nicht wissenschaftlich belegen [7].

\section{Expertinnenstandard}

Der von Hebammen entwickelte Expertinnenstandard zur „Förderung der physiologischen Geburt“ zeigt, wie physiologische Geburten möglichst ohne Einsatz von Interventionen gefördert werden können [9]. Mit Struktur-, Prozess- und Ergebniskriterien kann die Qualität der Geburtshilfe überprüft werden. Der Standard basiert auf einer systematischen Literaturrecherche und einem Konsens in der Expertinnengruppe (klinisch und außerklinisch tätige Hebammen, Kreißsaalleitungen, Hebammenlehrerinnen, Wissenschaftlerinnen). Er entspricht einem S1 Evidenzlevel, ohne dass er exakt in die Hierarchiestufen der medizinischen Leitlinien eingeordnet werden kann. Bis vor kurzem wurde die Anwendung des Expertinnenstandards in ausgewählten Kreißsälen erprobt. Einige Verantwortliche berichten von sinkenden Interventionsraten nach der Einführung. Eine wissenschaftliche Evaluation steht jedoch noch aus.

\section{Qualitäts- managementsysteme}

In den letzten Jahren haben viele Kliniken sogenannte Qualitätsmanagementsysteme eingeführt. Die „Kooperation für Transparenz und Qualität im Gesundheitswesen“ (KTQ) und die DIN EN ISO (Normenreihe $9000 \mathrm{ff}$ ) sind die gängigsten.

KTQ wurde von den Spitzenverbänden der gesetzlichen Krankenkassen und der Deutschen Krankenhausgesellschaft speziell für Krankenhäuser entwickelt [10]. Die Zertifizierung nach KTQ ist freiwillig. Sie erfolgt mit einer Selbstbewertung mithilfe eines Kriterienkataloges und mit einer Fremdbewertung durch Visitation. Insgesamt werden in diesem Prozess 72 Qualitätskriterien betrachtet. Ein Zertifikat wird für 3 Jahre vergeben, wenn mindestens 55\% der Maximalpunktzahl erreicht wird [11].

Auch die DIN EN ISO (Normenreihe 9000 ff) ist im Kliniksektor weit verbreitet [11]. Dieses Qualitätsmanagementsystem stammt aus der industriellen Fertigung und stellt lediglich einen Rahmen für die Ausgestaltung des Qualitätsmanagements dar. Die DIN EN ISO beinhaltet kaum konkrete Vorgaben und die Kliniken müssen diesen vorgegebenen Rahmen selbst mit Inhalt füllen. Das QMSystem besteht aus einem Katalog, der überwiegend organisatorische Prozesse beleuchtet und der von den Kliniken mit Prozessbeschreibungen und Kriterien für Qualität gefüllt wird. Das Ergebnis ist ein klinikspezifisches Qualitätshandbuch. Die ISO Norm wird bei einer Zertifizie-

\section{Definitionen}

Leitlinien sind systematisch entwickelte Therapie- und Entscheidungshilfen für Ärzte zu spezifischen Themen oder Krankheiten. Sie beruhen auf aktuellen wissenschaftlichen Erkenntnissen und Praxiserfahrungen [4].

„Die Expertenstandards sind Instrumente, mit deren Hilfe die Qualität von Pflegeleistungen definiert, realisiert und bewertet werden kann und die Auskunft darüber geben, welche Verantwortung die Berufsgruppe der Pflegenden [...] hat.“ [21]

„Ein Qualitätsindikator dient der Bewertung, ob ein Qualitätsziel erreicht wird.“ [23] „Er misst den Erfüllungsgrad von relevanten Qualitätsanforderungen.“ [22]

rung von einem externen autorisierten Gutachter mittels Visitation geprüft und gilt für drei Jahre [11].

Die Realisierung von Qualitätsmanagementsystemen ist mit einem hohen personellen und finanziellen Aufwand verbunden. Folgende Aspekte werden bei einigen Systemen kritisiert:

- Die Reliabilität und Validität der Qualitätsmessung ist in einigen Systemen problematisch: Es ist nicht garantiert, dass zwei Gutachterteams zum gleichen Ergebnis kommen.

- Die Ergebnisqualität der Krankenhäuser wird kaum geprüft.

- Qualität hat eine geringe Bedeutung in der täglichen Arbeit.

- Die Zertifizierungsverfahren selbst verfügen selbst nur über wenig konkretes Qualitätsmanagement.

- Es fehlen schlüssige Belege zur Kosteneffektivität der Zertifizierungsverfahren.

- Die Systeme regeln vor allem organisatorische Fragen. Fragen hinsichtlich einer patientennahen, medizinischen und pflegerischen Versorgung werden zu wenig thematisiert [12].

Trotz dieser Kritik berichten einige Kliniken in Erfahrungsberichten von positiven Auswirkungen. Der Prozess der Zertifizierung wird als wertschöpfend angesehen oder als Anstoß, längst Geplantes umzusetzen. Eine Zertifizierung sei nützlich, um Schwachstellen im Betrieb aufzudecken [13]. Auch interdisziplinäre Veränderungen gelängen besser. Als wichtigste Veränderungen durch Zertifizierungen gelten:

- Transparenz des Klinikgeschehens,

- höheres Qualitätsbewusstsein,

- steigende Mitarbeitermotivation,

- größere Identifikation der Mitarbeiterinnen/Mitarbeiter mit der Klinik,

- ausgeprägter Organisationsgrad wichtiger Abläufe und

- mehr Patientensicherheit [13].

Deutlich wird außerdem, dass zusätzliche Stellen, Engagement außerhalb der Arbeitszeit, ein zusätzlicher administrativer und ein hoher Kommunikationsauf- 
wand nötig waren [13]. Die Autoren fanden keine Belege für eine Verbesserung der Versorgungsqualität (im Sinne der Ergebnisqualität) durch eine Zertifizierung [13].

Neugebauer et al. untersuchten die Wirkung von KTQ und stellten folgende Verbesserungen fest:

- klare Definition von Zuständigkeiten und Neuregelung von Aufgabenspektren

- Veränderung des Führungsverhaltens

- Akzeptanz des Qualitätsmanagementsystems

• höhere Mitarbeiterzufriedenheit [14]

Schubert hat in ihrer Untersuchung 19 Krankenhäuser nach Aufwand und Nutzen eines Zertifizierungsverfahrens befragt. Sie stellte folgende Verbesserungen fest:

\section{Qualitätsindikatoren in der Geburtshilfe}

- E-E-Zeit (Zeit, die vom Entschluss zur Notsectio bis zur Entwicklung/Geburt des Kindes vergeht) bei Notsectio unter 20 Minuten

- Anwesenheit eines Pädiaters bei Frühgeburten

- Bestimmung des arteriellen Nabelschnur pH Wertes bei Geburten

- keine kindliche Azidosen bei Geburt $(\mathrm{pH}<7,00)$

- kein kritisches kindliches Outcome ( $\mathrm{pH}<7,00$ und 5-Minuten-Apgar $<5$ und Base Excess $\leq 16$ )

- möglichst kein Dammriss III oder IV

- durchgeführte Lungenreife bei Frühgeburten, wenn die Frauen vorher mindestens zwei Tage stationär behandelt wurden

- keine mütterlichen Todesfälle

- Antibiotikagabe bei vorzeitigem Blasensprung

- Antibiotikagabe bei Sectio

[19].
- größere Transparenz des Klinikgeschehens

- generelle Anhebung des Qualitätsbewusstseins

- höhere Motivation der Mitarbeiterinnen/Mitarbeiter

- höherer Organisationsgrad wichtiger Abläufe

- höhere Patientensicherheit [15]

Ernst et al. fanden in ihrer Untersuchung in einem Perinatalzentrum Level 1 nach einer Zertifizierung eine größere Transparenz, eine sehr gute interdisziplinäre Zusammenarbeit und eine frühzeitige Erkennung von Schwachstellen [16].

\section{Benchmarking und Qualitätsindikatoren}

Benchmarking stammt aus der Wirtschaft und ist ein kontinuierlicher Prozess, in dem die eigenen Leistungen mit den Leistungen anderer verglichen werden, und zwar derjenigen, die als überlegen angesehen werden. „Die Ergebnisse dieses Vergleichs werden qualitativ bewertet und führen so zur Implementierung und Anpassung von neuen Methoden und Prozessen (,Bestlösung‘)“ [17]. Es geht darum, besser als andere zu werden [17].

Die Wirkung von Benchmarking ist bereits mehrfach untersucht worden. Die Bereitstellung von Informationen aus dem Benchmarking steht in Zusammenhang mit stärkerer Motivation, Kosteneffizienz und Leistungsverbesserung. Die Wirkung wird mit der Theorie des sozialen Vergleichs erklärt. In dieser Theorie geht man davon aus, dass Menschen sich grundsätzlich mit anderen vergleichen möchten. Dadurch erfahren sie mehr über den eigenen Status, die eigenen persönlichen Fähigkeiten und können sich besser einschätzen. Menschen wollen geringfügig besser sein als andere und sind somit motiviert, die eigenen Anstrengungen zu intensivieren [18].

Im Sinne von Benchmarking können die Qualitätsindikatoren wirken. Qualitäts- indikatoren werden derzeit vom AquaInstitut evidenzbasiert entwickelt. Das Aqua-Institut ist eine fachlich unabhängige Institution, die im Rahmen eines Vergabeverfahrens ausgewählt wurde und die gemäß § 137a SGB V Verfahren zur Messung und Darstellung der Versorgungsqualität im Gesundheitswesen entwickelt.

Die bundesweiten Ergebnisse der jährlichen Erhebungen dieser Qualitätsindikatoren werden veröffentlicht und sind im Internet frei zugänglich. Somit haben alle geburtshilflichen Kliniken die Möglichkeit, ihre eigenen Ergebnisse mit dem Bundesdurchschnitt zu vergleichen und Konsequenzen abzuleiten. $\mathrm{Zu}$ jedem Qualitätsindikator ist ein erwünschter Zielbereich als Mindeststandard definiert, den die Kliniken möglichst erreichen sollten. Abb. 1 zeigt die Entwicklung in Deutschland seit Einführung der Qualitätsindikatoren.

Die meisten Ziele werden inzwischen erreicht. Lediglich die Antibiotikagabe bei vorzeitigem Blasensprung ist im Bundesdurchschnitt bei weniger als 95\% der Fälle erreicht und benötigt mehr Anstrengung.

Offenbar kann eine externe Kontrolle (durch das Aqua-Institut) die interne Qualitätsentwicklung (in den Kliniken) fördern. Besonders auffällig ist, dass eine E-E-Zeit von 20 Min. bei einer Notsectio kaum noch überschritten wird, dass es zunehmend gewährleistet ist, dass Pädiater bei Frühgeburten anwesend sind und dass vor Frühgeburten meistens eine Lungenreife verabreicht wird. Hier können große Qualitätssteigerungen nachgewiesen werden.

Die vorliegenden Indikatoren bilden nicht die gesamte geburtshilfliche Qualität ab. Wichtige Aspekte wie Wohlbefinden und Zufriedenheit der Frauen werden nicht berücksichtigt. 


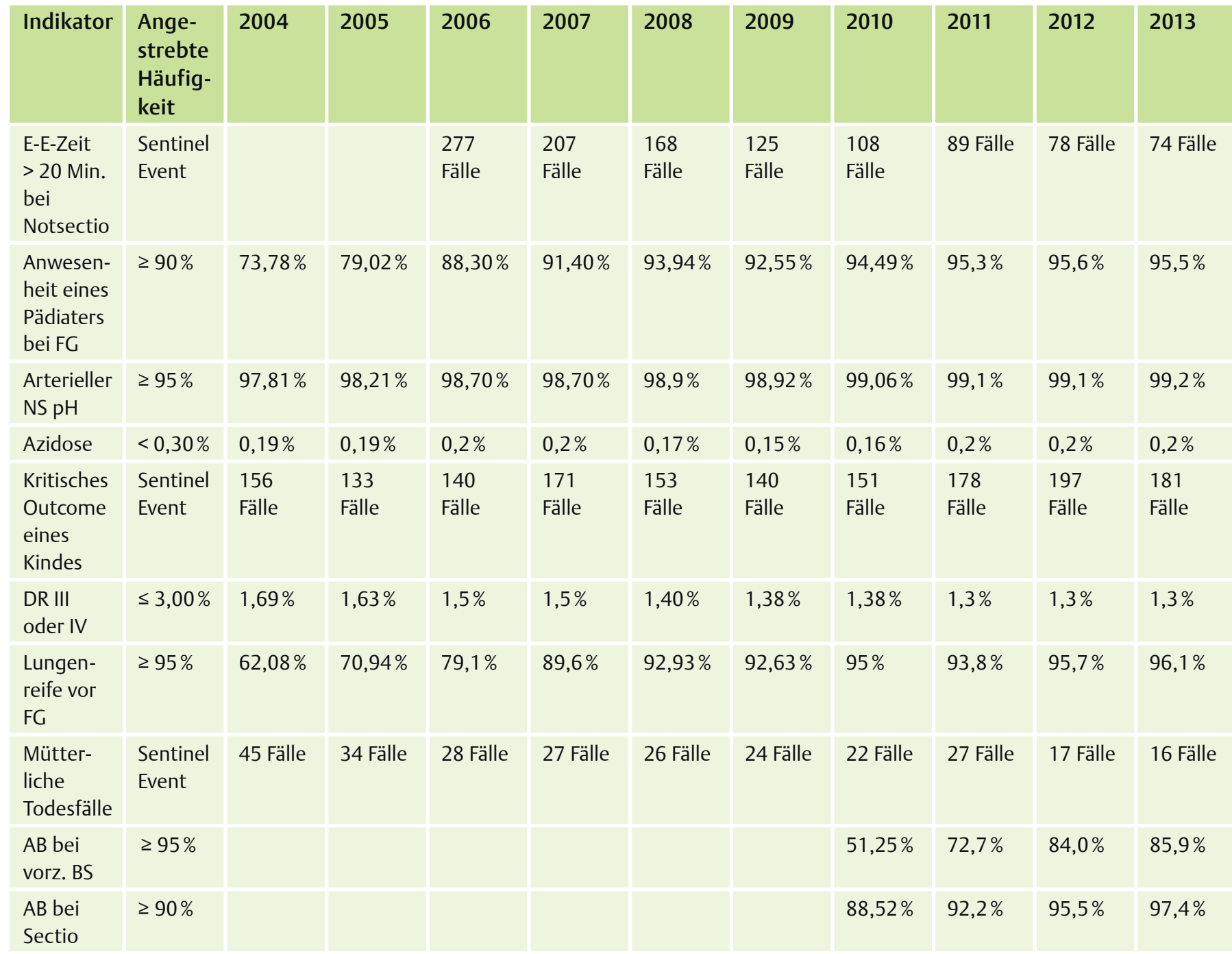

Sentinel Event: jeder Einzelfall stellt eine Auffälligkeit dar und muss analysiert werden

E-E-Zeit: Zeitraum, der zwischen Entscheidung zur Notsectio bis zur Entwicklung des Kindes vergeht FG: Frühgeburt; NS: Nabelschnur; AB: Antibiose; BS: Blasensprung

Abb. 1 Entwicklung der Qualitätsindikatoren über die vergangenen zehn Jahre in Deutschland (Eigenerstellung 2014 in Anlehnung an BQS 2004-2007 und AQUA 2008-2013).

\section{Fazit}

In den geburtshilflichen Kliniken werden zahlreiche Anstrengungen unternommen, um die Qualität zu sichern und zu verbessern. Jede Form von Qualitätsmanagement benötigt finanzielle, personelle und zeitliche Ressourcen sowie das Engagement der Beteiligten. Deshalb ist es erstrebenswert, dass Qualitätsmanagement nachweislich einen Nutzen für Frauen und Neugeborene hat und nicht lediglich dem Marketing einer Klinik dient. Hier besteht dringender Forschungsbedarf.
Um die Qualität in der Geburtshilfe umfassender zu messen, sind Qualitätskriterien und -ziele sinnvoll, die mit der Berufsgruppe der Hebammen abgestimmt sind bzw. hebammenwissenschaftliche Erkenntnisse beachten.

\section{Qualität außerhalb der Klinik}

Die Frage nach der Qualität wird längst auch in der freiberuflichen Hebammenarbeit gestellt. Seit 1996 werden außerklinische Geburten statistisch erhoben und zunächst auf freiwilliger Basis ausgewertet. 1999 gründete sich aus diesem Engagement die Gesellschaft für Qualität in der außerklinischen Geburtshilfe (QUAG e.V.). Die QUAG veröffentlicht jährliche Qualitätsberichte, um u.a. die Sicherheit für Mutter und Kind sowie die Kompetenz der Hebammen in der außerklinischen Geburtshilfe zu belegen. Seit 2008 übernehmen Krankenkassen die Betriebskostenpauschale für Geburtshäuser und verpflichtet diese zur Umsetzung eines Qualitätsmanagementverfahrens [20]. 
Ab 1.1.2015 müssen freiberufliche Hebammen ein Qualitätsmanagement nachweisen, um weiter mit den gesetzlichen Krankenkassen Leistungen abrechnen zu können. Hierfür stellt der DHV ein Qualitätsmanagementhandbuch in Anlehnung an die DIN EN ISO zur Verfügung. Diese Vorlage muss allerdings von den Anwenderinnen ,mit Leben gefüllt' werden.

\section{Literatur}

1 Bruhn M. Qualitätsmanagement für Dienstleistungen: Grundlagen, Konzepte, Methoden. 8., überarbeitete und erweiterte Auflage. Berlin: Springer, 2011

2 Selbmann H-K (2013). Braucht das deutsche Gesundheitssystem die neuen DIN EN 15224 Norm für das Qualitätsmanagement? In: Gesundheitsökonomie und Qualitätsmanagement 18., S. 142-143

3 Sackett D, Rosenberg W, Gray J, Haynes R, Richardson W (1997). Was ist Evidenz-basierte Medizin und was nicht? Übersetzung: Perleth, M. Medizinische Wochenschrift, 139, S. 644-645

4 AWMF (2014). Leitlinien. Verfügbar unter: http://www.awmf.org/leitlinien. html [28.10.2014]

5 ÄZQ (2007). Leitlinien-Glossar. Verfügbar unter: http://www.awmf.org/ fileadmin/user_upload/Leitlinien/ Werkzeuge/ll-glossar.pdf [28.10.2014]

6 Nothacker M, Kopp I (2013). Evidenzbasierte Indikationsstellung: Möglichkeiten und Grenzen von klinischen Studien und Leitlinien. Vortragsfolien vom Frühjahrsforum der Deutschen Hochschulmedizin, 18.04.2013, Verfügbar unter: http://www.awmf.org/ fileadmin/user_upload/Leitlinien/ AWMF-Publikationen/VUD_4_13mnf. pdf [28.10.2014]

7 Simoes E, Boukamp K, Mayer E, Schmahl $F$ (2004). Gibt es Belege für den Impact qualitätssichernder/-fördernder Verfahren in anderen Ländern? In: Das Gesundheitswesen, 66, S. 370-379

8 Knoll A (2010). Ist das routinemäßige Vorgehen in der Geburtshilfe in Deutschland leitlinienorientiert und evidenzbasiert? Verfügbar unter: http:// opus4.kobv.de/opus4-fau/frontdoor/ index/index/docId/1725 [03.11.2014].

\section{Internetadressen}

Aqua (2009-2013). Bundesauswertungen verfügbar unter: https://www.sqg.de/ ergebnisse/leistungsbereiche/geburtshilfe.html

BQS (2004-2007). Bundesauswertungen verfügbar unter: http://www.bqsqualitaetsindikatoren.de/archiv

9 DNQP, Verbund Hebammenforschung (2013). Expertinnenstandard Förderung der physiologischen Geburt. Osnabrück

10 Kahla-Witzsch H. Praxiswissen Qualitätsmanagement im Krankenhaus: Hilfen zur Vorbereitung und Umsetzung. 2. Auflage, Stuttgart: Kohlhammer, 2009

11 Denz C, Krieter H, v. Ackern K (2004). Stellenwert des Qualitätsmanagements und der Zertifizierung im Krankenhausbereich. In: Gesundheitsökonomie und Qualitätsmanagement (9), S. 362-391

12 Selbmann H-K (2004). Bewertung und Zertifizierung von Akut-Krankenhäusern in Deutschland. In: Bundesgesundheitsblätter, Gesundheitsforschung, Gesundheitsschutz, 2, S. 103-110

13 Vagts D, Bauer M, Martin J (2009). (Un) Sinn von Zertifizierungsmaßnahmen in der Intensivmedizin. In: Der Anaesthesist, 1, S. 81-87

14 Neugebauer F, Schiller M, Luger TA, Hensen P (2013). Evaluation einrichtungsinterner Effekte nach Durchführung eines Zertifizierungsverfahrens im Krankenhaus. In: Gesundheitswesen, 75, S. $108-112$

15 Schubert HJ (2006). Aufwand-/ Nutzen-Bewertungen zur Zertifizierung nach KTQ bzw. proCumCert Verfügbar unter: http://www.ktq.de/fileadmin/ media/Artikel/KTQ-Studie-Schubert.pdf [4.11.14]

16 Ernst T, Kiene P, Kämpf AK, Lange J, Grüßner S, Louwen F (2013). Wie viele Zertifizierungen brauchen wir noch? Wird die Qualität in Perinatalzentren Level I durch Zertifizierungen besser? In: Zeitschrift für Geburtshilfe und Neonatologie, 217 - V02_2, DOI: 10.1055/s-0033-1361193.

17 Bredl K, Hüsig S, Angele MK, Lüring C (2010). Strategisches Benchmarking. Eine potenzielle Antwort auf die raschen Veränderungen im Gesundheitswesen. In: Der Orthopäde. Verfügbar unter: http://link.springer. com/article/10.1007/s00132-010-16340/fulltext.html [30.10.14]
18 Mahlendorf MD, Kleinschmit F (2011). Reporting in deutschen Krankenhäusern - die Bedeutung von BenchmarkingInformationen. In: ZfCM Controlling \& Management, 55. Jg., H.4, S. 216-223

19 Aqua (2014). Geburtshilfe Indikatoren 2013. Verfügbar unter: https://www. sqg.de/downloads/QIDB/2013/ AQUA_16n1_Indikatoren_2013.pdf [5.11.2014]

20 QUAG. Verfügbar unter: www.quag.de [26.11.2014]

21 Wolke R, Allgeier C (2012). Expertenstandard Ernährungsmanagement. Nur Kosten oder auch Nutzen? Lage Jacobs

22 Aqua (2013). Allgemeine Methoden. Verfügbar unter: www. sqg.de

23 BQS (2009). Qualität sichtbar machen. Geschäftsbericht 2008. Düsseldorf, Eigenverlag

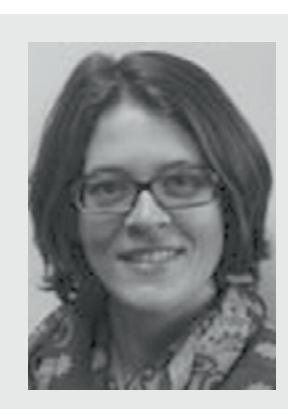

\section{Karin Schmidt}

Hebamme, B. A. Pflegepädagogik, M. A. Pflegewissenschaft, Lehrerin an der Hebammenschule Stuttgart Schlößlestr. 5 70186 Stuttgart E-Mail: Sch-karin@web.de 